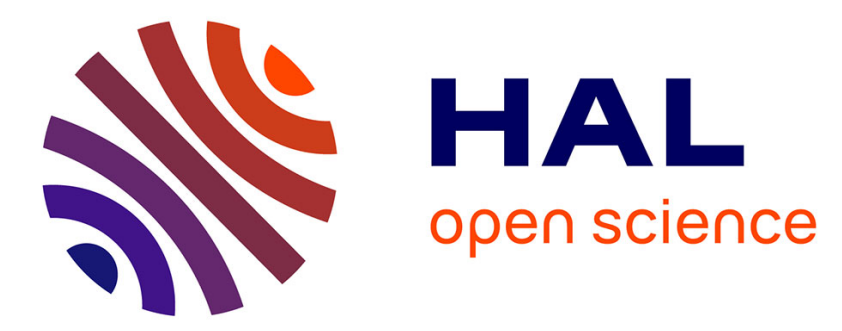

\title{
Potentiel relationnel et impact du site de marque sur la performance marketing
}

Pierre Volle, Laurent Florès

\section{To cite this version:}

Pierre Volle, Laurent Florès. Potentiel relationnel et impact du site de marque sur la performance marketing. Décisions Marketing, 2005, 40, pp.39-50. halshs-00151273

\section{HAL Id: halshs-00151273 \\ https://shs.hal.science/halshs-00151273}

Submitted on 23 Jul 2007

HAL is a multi-disciplinary open access archive for the deposit and dissemination of scientific research documents, whether they are published or not. The documents may come from teaching and research institutions in France or abroad, or from public or private research centers.
L'archive ouverte pluridisciplinaire HAL, est destinée au dépôt et à la diffusion de documents scientifiques de niveau recherche, publiés ou non, émanant des établissements d'enseignement et de recherche français ou étrangers, des laboratoires publics ou privés. 


\title{
Potentiel relationnel et impact du site de marque sur la performance marketing
}

\author{
Laurent Florès \\ CEO, CRMMetrix \\ Pierre Volle \\ Professeur de Marketing, Université Paris Dauphine \\ Dauphine Recherches en Management - CNRS, UMR 7088 \\ Professeur affilié, ESCEM
}

Résumé : L’analyse d’une base de données composée de 102 études réalisées en ligne montre que le site de marque est un outil marketing qui permet d'améliorer plusieurs indicateurs de performance - notamment l'intention d'achat et l'attitude vis-à-vis de la marque - et permet d'engager un échange avec les meilleurs clients. Nous formulons plusieurs recommandations pour améliorer la performance marketing et le potentiel relationnel des sites de marque.

Mot clés : marketing relationnel, sites de marque, performance marketing

\begin{abstract}
Based on 102 online surveys accounting for more than 3 million interviews, we show that websites are powerful purchase intent and brand builders and represent a strong opportunity to enhance customer relationships through “conversation” with the brand's best customers.
\end{abstract}

Key words: relationship marketing, brand websites, marketing performance

Présentation des auteurs: Laurent Florès est fondateur et CEO de CRMMetrix (www.crmmetrix.com), société spécialisée dans l'écoute client sur Internet. Il s'intéresse au développement de nouvelles méthodes de « conversations » entre consommateurs et marques, au marketing relationnel et à l'innovation. Pierre Volle est Professeur de marketing à l'Université Paris Dauphine, Directeur de recherche au DMSP (Centre de recherche en Marketing, Stratégie, Prospective), Responsable du Master Distribution \& Relation Client et Professeur affilié à l'ESCEM, en charge d'activités de recherche sur le marketing relationnel. Il s’intéresse à l'étude des consommateurs, aux enjeux du marketing relationnel et à la mise en œuvre opérationnelle de stratégies client. 


\section{Potentiel relationnel et impact du site de marque sur la performance marketing}

Une présence des marques sur le Net semble aujourd'hui incontournable. D’après une enquête en ligne menée en 2004 par le Journal du Net auprès de 536 internautes français, 70 \% des répondants estiment qu'une marque sans site Internet "se désintéresse de ses clients » (source : www.journaldunet.com). Dans une enquête menée en mai 2003 auprès de 2123 internautes américains, le site de marque se place très loin devant le courrier électronique ou les formes de communication plus intrusives pour " apprendre et échanger » avec ses marques favorites : 55\% des internautes déclarent préférer le site de marque, contre $22 \%$ le courrier électronique, $9 \%$ la publicité TV, $6 \%$ la publicité en ligne, 5\% le courrier adressé et $4 \%$ la publicité presse ou magazine (source : $\underline{w w w . c r m m e t r i x . c o m)}$ ).

Si le site de marque pouvait initialement être considéré comme un canal marketing généraliste avec des objectifs de notoriété et de vente, on peut aujourd'hui l'appréhender comme un outil permettant de créer et de maintenir des relations fortes avec les meilleurs clients, à l'instar d’Henkel qui présente sa stratégie de site de la façon suivante: «Nous avons choisi de communiquer de façon plus subtile et plus intelligente que par une promotion agressive autour de nos produits. C'est pourquoi nous avons crée un site de conseils et d'astuces pratiques, afin d'établir une relation de proximité avec le client final ».

La demande de contenus informatifs est très forte de la part des clients ou futurs clients. L'enquête menée par le Journal du Net montre que la recherche d'information constitue la première motivation pour fréquenter les sites de marque (78\%), devant les conseils d'utilisation et d'entretien (51\%), la demande de contact avec le SAV (28\%) ou encore, la participation à des jeux-concours (24\%). Sachant que les consommateurs s'informent de plus en plus en ligne - c'est le cas, par exemple, dans l'automobile où près de $70 \%$ des futurs acheteurs américains visitent les sites des constructeurs avant d'acheter (7) - le site doit devenir une source d'information crédible et reconnue au niveau de sa catégorie.

L'exemple du site Kraftfoods.com est, à cet égard, emblématique. En quelques années, le site est passé d'un simple site à vocation informationnelle à un véritable site à vocation relationnelle devenant la référence en matière de conseils cuisine, santé et bien-être, ne cessant de personnaliser les messages diffusés à ses visiteurs enregistrés durant leurs visites et dans leur boite aux lettres électroniques (la fréquence d'envoi des recettes étant désormais hebdomadaire). 
Dans cette même perspective, l'exemple du site Tide.com (Ariel en France) est également intéressant, pour une catégorie sans doute moins impliquante que celle de l'alimentaire. La marque s'efforce d'apporter des services à ses membres et ses visiteurs via, par exemple, le « Stain Detective » qui apporte des conseils pour enlever les tâches selon leur nature et le tissu sur lequel elles ont été faites. Au-delà de ces services, le site est également très performant pour relayer des opérations promotionnelles.

Les sites de marque sont relativement onéreux à concevoir et à animer, avec des budgets compris entre 100000 et 500000 \$ (source : www.forrester.com). Cependant, comme nous allons tenter de le montrer dans cet article, cet investissement peut se révéler judicieux dans la mesure où le site peut permettre de toucher ses clients réguliers et d'améliorer encore les indicateurs de performance marketing, comme l'attitude vis-à-vis de la marque.

En ce qui concerne les sites de marque (Tableau 1), précisons que nos analyses portent sur les sites relationnels et sur les mini-sites promotionnels, c'est-à-dire, les sites «conçus pour enrichir l'image et la notoriété d'une marque, ou pour renforcer sa relation aux clients, qu'ils soient des réalisations pérennes, ou qu’ils aient été conçus pour ne rester en ligne que quelques mois », comme les sites liés à des lancements de produits ou à de grandes opérations promotionnelles (source : wwww.toutsurlacom.com). Par ailleurs, précisons également que nos analyses concernent uniquement des sites de marque à destination des adultes et non à destination des enfants, cette dernière cible ayant des motivations et des comportements très spécifiques (3).

\section{< Insérer le tableau 1 >}

Les résultats développés dans l'article reposent sur l'exploitation d'une base de données d'études gérée par CRMMetrix, institut d’études marketing spécialisé dans l'écoute client sur Internet (Encadré 1). Cette base contient plus de 3 millions d'interviews en ligne réalisées entre 2001 et 2004 à partir de plusieurs sites de marque issus de secteurs divers (produits de grande consommation, équipement, automobile, etc.), en Europe et aux Etats-Unis. La base est formée de l'agrégation de 102 études (data pooling) toutes menées sur la même version du site, durant un trimestre avec un tirage aléatoire de 30000 observations pour chaque étude. Cependant, si nos analyses reposent essentiellement sur la base de données SiteCRM de CRMMetrix, nous mettons également en perspective les principaux résultats des rares recherches académiques publiées à ce jour à propos des sites de marque.

< Insérer l’encadré 1 > 
L'analyse permet d'aboutir à trois principaux résultats qui sont développés par la suite :

- le site de marque permet d'identifier une part importante des meilleurs clients ; il s'agit de valoriser le site de marque comme un outil destiné à renforcer les relations avec les clients réguliers;

- la visite du site de marque a un impact non négligeable sur l'intention d'achat et sur l'attitude vis-à-vis de la marque; il s'agit de faire en sorte que le site de marque devienne un point de contact privilégié;

- le principal facteur qui semble déclencher l’intention de revisiter le site est la qualité de l'expérience en ligne ; pour le responsable de site, il s'agit avant tout de proposer un contenu valorisé par la cible.

\section{Le site de marque permet d'identifier une part importante des meilleurs clients}

Les études d'audience sur Internet montrent qu'aucun site de marque ne se glisse parmi les 20 premiers sites - en nombre de visiteurs uniques (source: www.netratings.com) - les premières places étant occupées par des portails et par des sites marchands comme Amazon.com ou Ebay.com. Les sites de marque, y compris les plus connus, n’atteindrons probablement jamais l'audience des sites de recherche ou des portails. Si le média-planning pour les médias de masse vise usuellement à créer une exposition maximale, sous contrainte d’une répétition adéquate, quant est-il pour les sites de marque?

L'analyse de la base de données SiteCRM révèle que si le site de marque permet, en moyenne, de toucher moins de $0,5 \%$ de la population totale, ce qui constitue effectivement une faible couverture, celui-ci permet en revanche de toucher 15\% des meilleurs clients de la marque, sachant que $70 \%$ du trafic au site est constitué d'acheteurs de la marque, dont $60 \%$ d'acheteurs réguliers (Figure 1). Comparativement aux médias de masse qui permettent des millions de contacts, dont une grande majorité auprès de non clients, le site de marque apparaît donc plutôt comme un outil pour attirer les meilleurs clients, y compris les leaders d'opinion (13).

< Insérer la figure 1 > 
A l'heure où les responsables de site sont de plus en plus attentifs au rendement des investissements Internet, la perspective de s'adresser, via les sites de marque, aux clients qui représentent entre 10 et $15 \%$ du chiffre d'affaires est naturellement motivante ${ }^{1}$.

Pour toucher des clients potentiels, au-delà de ce premier cercle des meilleurs clients, le responsable du site de marque peut également bénéficier d'un flux de communication naturel entre internautes, qui présente de nombreuses qualités : faible coût, degré de ciblage élevé et forte crédibilité (4). Sans incitation particulière - comme les offres de parrainage - cette communication naturelle entre internautes est déjà élevée. Ainsi, les données issues de SiteCRM montrent que le pourcentage de visiteurs satisfaits qui se disent prêts à recommander le site s'élève à $61 \%$. Ce pourcentage est encore plus élevé pour les visiteurs réguliers (visiteurs ayant déjà visité le site), puisqu’il s’élève à 73\%.

En synthèse, le site de marque n’est donc pas un outil marketing destiné à recruter des clients ou à toucher un large public indifférencié, mais plutôt à identifier les meilleurs clients de la marque. Par ailleurs, l'intérêt des sites de marque est renforcé lorsque l'on cible les clients susceptibles de relayer les messages auprès de leurs pairs.

\section{La visite du site de marque a un impact significatif sur l'intention d'achat et sur l'attitude vis-à-vis de la marque}

La présence de la marque sur Internet peut influencer les comportements ultérieurs des visiteurs à l'égard de l'entreprise et de ses produits. Ce résultat a notamment été montré pour des sites marchands, dans la mesure où la satisfaction du visiteur vis-à-vis du site a des incidences sur son degré de fidélité off line à l'égard de l'enseigne (15). Cependant, ce résultat est-il également valable pour les sites de marque?

Pour étudier cette question, un échantillon de contrôle de visiteurs - pour lequel la mesure de l'intention d'achat est effectuée en début de visite - a été comparé à un échantillon test - pour lequel la mesure est effectuée en "sortie de site » (ces deux échantillons sont constitués de visiteurs qui se rendent sur le site pour la première fois). Sur l'ensemble de la base SiteCRM, l'augmentation de l'intention d'achat consécutive à la visite se situe autour de 5\% (Tableau 2).

Ceci dit, cette augmentation moyenne masque de fortes disparités. En effet, si l'augmentation peut atteindre $60 \%$ pour certains sites, on peut également isoler des impacts négatifs, quand l'expérience du site est jugée très défavorablement.

< Insérer le tableau 2 >

\footnotetext{
${ }^{1}$ Si l'on considère que $20 \%$ des clients réalisent $80 \%$ du chiffre d'affaires, la possibilité de toucher $16 \%$ des meilleurs clients via le site de marque, permet donc de peser sur $16 \%$ x $80 \%=13 \%$ du CA.
} 
Des analyses complémentaires montrent que l'impact est plus fort pour les marques dont la notoriété est plus faible. En effet, si l'impact moyen de la visite sur l'intention d'achat se situe autour de $5 \%$, il monte à $5-7 \%$ pour les marques moins connues (versus $2-3 \%$ pour les marques les plus connues).

Au-delà de l'intention d'achat, la visite d'un site est-elle susceptible d'influencer l'attitude vis-à-vis de la marque ? En effet, si les marques s'incarnent aujourd'hui de façons très diverses - produits, packaging, messages publicitaires, personnel en contact... - l'expérience totale de la marque repose probablement en partie sur l'expérience du site. Une mauvaise expérience peut avoir des incidences négatives sur la perception de la marque et vice-versa. Sur ce point, les données de la base SiteCRM montrent que plus l'expérience en ligne est jugée positivement par l'internaute, plus son attitude à l'égard de la marque s'améliore (Tableau 3). Près de $50 \%$ des visiteurs très satisfaits de leur expérience sur le site révisent favorablement leur attitude vis-à-vis de la marque. Ce résultat est identique pour les visiteurs réguliers. Notons, cependant, qu'environ la moitié des visiteurs ne changent pas leur attitude à l'égard de la marque, suite à une simple visite, ce qui laisse supposer que l'effet du site de marque n’est en aucun cas systématique et homogène.

\section{$<$ Insérer le tableau $3>$}

Nos résultats montrent également que l'impact de la visite sur l'attitude vis-à-vis de la marque peut varier d'un type de produit à l'autre (produits alimentaires et boisson versus produits d'entretien), même si la simple visite bénéficie à la marque dans les deux configurations (Tableau 4a et 4b).

\section{$<$ Insérer les tableaux 4a et 4b >}

Concernant l'impact d'une visite sur la perception de la marque, ces résultats vont dans le même sens que ceux de Müller et Chandon (9). Cette recherche, l'une des rares à porter sur les sites de marque, montre qu'une attitude favorable vis-à-vis du site influence positivement la façon dont la marque est jugée en terme de jeunesse / modernité et de sincérité / confiance (tous les traits de personnalité de la marque ne sont pas influencés par la visite du site). Cette même recherche attire également l'attention sur des différences d'impact d'un type de produit à l'autre (produits fonctionnels comme l'électro-ménager, versus non fonctionnels comme le luxe).

En synthèse, si la visite d'un site de marque influence significativement des indicateurs de performance marketing importants - comme l'intention d'achat ou l'attitude vis-à-vis de la marque - un impact positif n'est pas assuré pour tous les sites (selon le niveau de notoriété initial ou la catégorie de produit, par exemple). Ces résultats premiers, pour intéressants qu'ils soient, nécessitent cependant de mieux comprendre quels sont les éléments qui déterminent plus précisément l'efficacité des sites de marque. 


\section{Le principal facteur qui semble déclencher l'intention de revisiter le site est la qualité de l’expérience en ligne}

La transformation des visiteurs occasionnels en visiteurs réguliers repose sur de nombreux facteurs, désormais assez bien identifiés dans la littérature relative aux sites marchands (Encadré 2). Toutefois, comme il n'existe pas, à notre connaissance, d'étude spécifique sur la fidélité aux sites de marque, dans quelle mesure les études menées sur les sites marchands peuvent-elle aider à comprendre ce phénomène ?

\section{< Insérer l'encadré 2 >}

L'une des principales questions aujourd'hui étudiée par les chercheurs concerne le lien entre la satisfaction et la fidélité. Ainsi, une recherche menée dans l’industrie hôtelière montre que le lien entre satisfaction et fidélité est plus fort en ligne: pour un niveau de satisfaction identique, le niveau fidélité sera plus élevé en ligne (11). Cette recherche montre également que la fidélité, en retour, a un impact sur la satisfaction et que ce renforcement réciproque est plus fort pour les services en ligne. Autrement dit, le cercle vertueux « satisfaction $\mathrm{t}_{\mathrm{t}-1} \rightarrow$ fidélité $_{t} \rightarrow$ satisfaction $_{t+1}$ » est encore plus critique pour le monde de l'Internet. Mais ce résultat est-il valable pour les sites de marque ? Quelle importance finalement accorder à la satisfaction pour expliquer l'intention de visiter à nouveau le site de marque ?

Sur cette question, les données issues de SiteCRM montrent effectivement que plus l'expérience en ligne est jugée satisfaisante, plus l'intention de visite est forte (Tableau 5). Ce résultat est encore plus net auprès des internautes qui ne visitent pas le site pour la première fois. Ainsi, le pourcentage de visiteurs «très satisfaits » qui ont l'intention «très probable " de visiter à nouveau le site augmente de $68,2 \%$ à $90,5 \%$.

\section{< Insérer le tableau 5 >}

Si le niveau de satisfaction du visiteur détermine en grande partie l'intention de revisiter le site, la question se pose, en amont, de savoir quels sont les éléments les plus susceptibles d'induire une expérience en ligne satisfaisante. Dans une recherche récente à propos de sites marchands, l'expérience en ligne - composée des trois éléments que sont la facilité de navigation, la qualité du contenu et le niveau de service - détermine en grande partie la valeur de la visite, autant que la qualité des produits et que les prix (1).

Concernant spécifiquement les sites de marque, les données de SiteCRM montrent que la satisfaction vis-à-vis du site de marque est liée significativement à la qualité du contenu (Tableau 6a), l'autre élément déterminant étant la facilité de navigation (Tableau 6b). De plus, l'impact de la qualité du contenu sur la qualité de l'expérience en ligne est, selon les cas, de 10 à 20\% supérieure à celle de la facilité de navigation sur le site (6). 
Pour affiner le propos, concernant la facilité de navigation, nous constatons que seuls les très hauts niveaux de satisfaction créent réellement une différence (effet non linéaire). On notera également que l'impact de ces facteurs - particulièrement la navigation - n'est pas le même sur le niveau de satisfaction et sur le niveau d'insatisfaction, un phénomène bien connu (8) qui consiste à dire que les facteurs qui concourent à la satisfaction ne sont pas nécessairement les mêmes que ceux qui conduisent à l'insatisfaction (effet non symétrique). Dans notre cas, le lien est plus net avec la satisfaction qu'avec l'insatisfaction.

$<$ Insérer les tableaux 6a et 6b >

Le niveau de satisfaction dépend également du comportement d'achat usuel du visiteur : les consommateurs ayant une forte affinité avec la marque (i.e., achat exclusif) sont significativement plus exigeants que les autres ${ }^{2}$. Sur ce point, les données montrent que l'importance donnée par le visiteur au contenu délivré sur le site augmente de 42,2\% pour les clients ayant une faible affinité à 58,3\% pour ceux qui ont une affinité forte.

La pression est donc forte sur les responsables de site : tous les points de contact doivent apporter de la valeur à l'expérience totale de la marque (15). Si l'on prend l'exemple de Disney, le site doit donner vie à l'univers magique de la marque, le même que celui des films ou des parcs. Si le site ne permet pas de vivre cette expérience «extra-ordinaire », il est probable que le visiteur ne sera pas satisfait. En retour, une expérience décevante a de fortes chances de rejaillir sur la marque elle-même. Cet « effet de transposition des attentes » d'un canal à l'autre constitue naturellement une contrainte incontournable pour qui prétend améliorer le niveau de satisfaction.

En synthèse, la fidélité du visiteur au site de marque dépend étroitement du niveau de satisfaction à l'égard du contenu offert sur le site. Les responsables de site doivent donc faire un effort particulier sur le contenu, qui ne doit pas simplement constituer un une réplique de brochure d'information. Ceci est particulièrement vrai auprès des clients réguliers de la marque, dont le niveau d'exigence est supérieur aux autres visiteurs.

\section{Quelques conclusions intermédiaires}

Les trois constats empiriques développés plus haut à partir de la base de données d'études de CRMMetrix permettent de penser que le site des marque est un outil efficace pour attirer un trafic ciblé et pour induire une amélioration de la performance, sous réserve que le contenu proposé sur le site soit à la hauteur des attentes, notamment informationnelles, des visiteurs.

\footnotetext{
${ }^{2}$ L’affinité avec la marque est mesurée de la façon suivante : «Quelle est parmi la phrase suivante celle qui correspond le mieux à votre relation à la marque $\mathrm{X}$ ? ». L’affinité forte correspond à la réponse « C'est la seule marque de lessive que j'achète » alors que l'affinité moyenne correspond à « $\mathrm{X}$ fait parti des deux ou trois marques de lessive que j’achète régulièrement». Les cinq autres positions de l'échelle sont agrégées pour représenter une affinité faible.
} 
Cependant, le site de marque ne conduit pas mécaniquement à une amélioration de la performance. Les analyses que nous avons menées montrent que cette performance marketing varie selon le type de produit (niveau de notoriété de la marque et catégorie de produit) et selon le type de client (niveau de satisfaction à l'égard du site et degré d'affinité préalable avec la marque).

Au-delà des investigations exploratoires que nous avons réalisées relativement à la performance transactionnelle des sites de marque (i.e., impact sur l'intention d'achat ou sur le degré de considération), cet outil marketing offre certainement un potentiel relationnel qu'il convient d'étudier plus en profondeur. Il est important d'identifier les conditions sous lesquelles ce potentiel pourrait, ou non, se révéler.

\section{Le site de marque, outil relationnel ?}

Voilà cinq ans, Internet était avant tout considéré comme un canal de vente : les experts promettaient aux marques qu'elles allaient vendre directement au consommateur final, pour contourner le distributeur. Comme on le sait, cette vision n'a pas encore pris corps, car elle sous-estimait largement les difficultés de mise en œuvre (14). En revanche, le potentiel relationnel d'Internet était à peine évoqué. S'il est probable que, dans cinq ans, ce potentiel apparaîtra comme une évidence il n’en reste pas moins qu'aujourd’hui, il ne nous semble pas pleinement exploité par les marques. L'utilisation d'indicateurs de performance comme le nombre de visiteurs uniques ou le chiffre d'affaires généré, pour mesurer le succès d'un site, révèle que la présence sur Internet est encore bien souvent assimilée à un média classique ou à un magasin ; le site de marque est trop rarement conçu comme un canal relationnel.

\section{< Insérer l’encadré 3 >}

Cependant, pour faire du site de marque un canal relationnel, il est indispensable d'engager une «conversation » avec les visiteurs. Il s'agit moins d'études marketing ponctuelles que d'une construction progressive de la connaissance client, à travers un dialogue continu. Cette conversation présente une valeur en soi - elle renforce le lien avec la marque, comme une conversation entre amis - et permet également d'éclairer le décideur sur de nombreux plans, comme la communication ou l'innovation.

Il est notamment utile d'identifier les leaders d'opinion, voire les «lead users », de façon à développer une relation spécifique avec eux. Il est ensuite souhaitable d’intégrer ces utilisateurs dans le processus de développement, une démarche qui augmente considérablement les chances de succès des nouveaux produits (2) grâce à de véritables communautés de consommateurs, mais aussi à des fins de marketing viral (5). Sur ces démarches d'implication des leaders d'opinion, on notera, par exemple, l'initiative de Procter \& Gamble avec la communauté de consommateurs Tremor (source : www.tremor.com). 
Pour mettre en place cette démarche relationnelle, l'une des premières problématiques concrètes auquel le responsable de site est confronté consiste à proposer au visiteur un processus d'enregistrement adéquat, de façon à pouvoir le reconnaître ultérieurement. Il s’agit d'un passage quasiment obligé pour pouvoir enclencher une relation plus personnalisée ${ }^{3}$. Cependant, si la plupart des sites proposent un tel processus d'enregistrement, il est le plus souvent jugé trop complexe par les internautes, ce qui les conduit à abandonner ou à vivre une expérience particulièrement frustrante.

Un questionnaire très court proposé dès la page d'accueil (en entrée ou en sortie) peut permettre de connaître les raisons qui ont amené le visiteur à venir sur le site, ce que le visiteur représente pour la marque (acheteur, client régulier...) et quel média permet d’attirer le trafic le plus ciblé : ou placer des bannières, quels liens promotionnels activer, quels sites partenaires privilégier, quelle importance donner au packaging ou aux publicités TV pour relayer le nom du site, etc. ? Dans un premier temps, la capture de l'adresse électronique peut même convenir pour commencer à cultiver la relation : à un stade préliminaire, il ne semble pas utile de décourager la bonne volonté du visiteur en exigeant d'emblée une trop grande masse d'informations.

Contrairement à ce que l'on pense bien souvent, les consommateurs sont plutôt disposés à donner leur avis sur les produits de leurs marques favorites, à parler de ce qu'ils aimeraient acheter, à comparer ces marques à leurs concurrents, à donner leur point de vue sur des nouveaux produits, etc. Les données de SiteCRM révèlent que le taux de participation aux enquêtes en ligne se situe entre $30 \%$ et $40 \%$ (on se souvient qu'en moyenne $60 \%$ des visiteurs sont des clients réguliers de la marque). Par ailleurs, près de $80 \%$ des visiteurs complètent le questionnaire jusqu'au bout et environ $90 \%$ des répondants estiment qu'il ne s'agit «pas d'une perte de temps » et qu'ils seraient "prêts à remplir à nouveau un questionnaire en ligne ». C'est ce dernier point qui permet en particulier d'envisager la mise en place d'échanges entre marque et consommateurs (5).

La mise en place d'une telle démarche de dialogue nécessite des changements importants chez l'annonceur. Le passage à une logique d'échange basée sur la "permission » nécessite une remise en cause du modèle du marketing traditionnel basé sur l'interruption, qui peut contrecarrer le potentiel relationnel dont dispose le site de marque. Il est particulièrement important de garder cette mise en garde à l'esprit, au risque de voir les clients rejeter toute tentative de « prise de contrôle » de la marque.

\footnotetext{
${ }^{3}$ On ne parle pas ici des cookies qui ne traduisent pas une volonté de rentrer en relation, mais plutôt le souci de pouvoir suivre l'individu d'une visite à l'autre, notamment à des fins de scoring, sans l'obtention d'une permission préalable, consciente et volontaire.
} 


\section{Références}

(1) Chen Z. et Dubinsky A.J. (2003), A conceptual model of perceived customer value in ecommerce, Psychology and Marketing, 20, 4, 323-47.

(2) Dahan E. et Hauser J.R. (2001), The virtual customer, Journal of Product Innovation Management, 19, 5, 332-354.

(3) De Lassus C. (2003), Les enfants et Internet : les freins et les motivations à l'égard du Web, Décisions Marketing, 31.

(4) Dholakia U.M., Bagozzi R.P. et Pearo L.K. (2004), A social influence model of consumer participation in network- and small-group-based virtual communities, International Journal of Research in Marketing, 21, 3.

(5) Figallo, C. (1998), Hosting web communities : building relationships, increasing customer loyalty, and maintaining a competitive edge, New York, Wiley Computer Publishing.

(6) Florès L. (2004), Measuring the sales impact of brand websites, Admap, October, 78-79.

(7) Klein L.R. et Ford G.T. (2003), Consumer search for information in the digital age : an empirical study of prepurchase search for automobiles, Journal of Interactive Marketing, 17, 3, 29.

(8) Lichtlé M.-C., Llosa, S. et Plichon V. (2002), La contribution des différents éléments d'une grande surface alimentaire à la satisfaction du client, Recherche et Applications en Marketing, 17, 4, 23-34.

(9) Müller B. et Chandon J.L. (2002), L'impact de la visite d'un site Internet sur la personnalité de la marque, Actes du Congrès International de l'Association Française du Marketing, Lille.

(10) Reibstein D.J. (2002), What attracts customers to online stores, and what keeps them coming back?, Journal of the Academy of Marketing Science, 30, 4, 465.

(11) Shankar V., Smith A.K. et Rangaswamy A. (2003), Customer satisfaction and loyalty in online and offline environments, International Journal of Research in Marketing, 20, 153-75.

(12) Srinivasan S.S., Anderson R. et Ponnavolu K. (2002), Customer loyalty in e-commerce : an exploration of its antecedents and consequences, Journal of Retailing, 78, 41-50.

(13) Vernette E. et Florès L. (2004), Communiquer avec les leaders d'opinion en marketing : comment et dans quels médias ?, Décisions Marketing, 35, 23-38.

(14) Volle P. (2000), Du marketing des points de vente à celui des sites marchands : Spécificités, opportunités et questions de recherche, Revue Française du Marketing, 177/178, 2-3, 83-101.

(15) Wallace D.W., Gieseb J.L. et Johnson J.L. (2004), Customer retailer loyalty in the context of multiple channel strategies, Journal of Retailing, 80, 78-96. 
Tableau 1 - Une typologie des sites de marques

\begin{tabular}{|c|c|c|}
\hline Type de site & Caractéristiques & Exemple \\
\hline Site corporate & $\begin{array}{l}\text { Présentation de l'entreprise pour l'ensemble des } \\
\text { parties-prenantes, particulièrement les } \\
\text { investisseurs et les futurs collaborateurs. } \\
\text { Contenu : valeurs de l'entreprise, communiqués } \\
\text { de presse, rapports annuels, etc. }\end{array}$ & Bongrain.com \\
\hline Site relationnel & $\begin{array}{l}\text { Présentation des l'ensemble des produits } \\
\text { proposés par l'entreprise (multi-marques), autour } \\
\text { d'un thème fédérateur (la maison, la santé, etc.). } \\
\text { Contenu : quizz, jeux, informations, guides, } \\
\text { accès au programme de fidélisation, etc. }\end{array}$ & $\begin{array}{l}\text { «Danone Conseils », } \\
\text { «Tout coller » de Henkel, } \\
\text { « La vie simplifiée » de } \\
\text { Procter \& Gamble }\end{array}$ \\
\hline $\begin{array}{l}\text { Mini-site } \\
\text { promotionnel }\end{array}$ & $\begin{array}{l}\text { Site relayant une campagne publi-promotionnelle } \\
\text { de grande envergure, à l'occasion d'un } \\
\text { lancement, d'un changement d'identité, d'un } \\
\text { anniversaire majeur, etc. Contenu : jeux, visuels } \\
\text { et spots publicitaires, etc. }\end{array}$ & $\begin{array}{l}\text { "Rêves d'enfant » à } \\
\text { l'occasion des } 40 \text { ans de } \\
\text { Nutella. Campagne } \\
\text { "Toutes les beautés » de } \\
\text { Dove }\end{array}$ \\
\hline Site tribal & $\begin{array}{l}\text { Variante du site relationnel et du mini-site dédiée } \\
\text { à un segment de clients particuliers voire, à une } \\
\text { tribu. Contenu : communication plus horizontale } \\
\text { entre clients, offres spécifiques }\end{array}$ & $\begin{array}{l}\text { Tremor.com (site de } \\
\text { recrutement de clients } \\
\text { leaders d'opinion) }\end{array}$ \\
\hline Site marchand & Site proposant des produits à la vente & Lancome.fr \\
\hline
\end{tabular}


Tableau 2 - L'intention d'achat avant / après la visite du site

\begin{tabular}{|l|c|c|}
\cline { 2 - 3 } \multicolumn{1}{c|}{} & $\begin{array}{c}\text { Mesure d'intention } \\
\text { d'achat en entrée de site }\end{array}$ & $\begin{array}{c}\text { Mesure d'intention } \\
\text { d'achat en sortie de site }\end{array}$ \\
\hline Très peu probable & $0,6 \%$ & $0,5 \%$ \\
\hline Peu probable & $1,0 \%$ & $0,9 \%$ \\
\hline Neutre & $13,9 \%$ & $6,2 \%$ \\
\hline Probable & $24,9 \%$ & $17,1 \%$ \\
\hline Très probable & $\mathbf{5 9 , 6 \%}$ & $\mathbf{7 5 , 2 \%}$ \\
\hline
\end{tabular}

Note : Test du chi-deux significatif à $p<0,001$ 
Tableau 3 - Lien entre la satisfaction vis-à-vis de l'expérience de visite et l'attitude à l'égard de la marque (internautes visitant le site de marque pour la $1^{\text {ière }}$ fois)

\begin{tabular}{|l|l|c|c|c|c|c|}
\cline { 3 - 7 } \multicolumn{2}{c|}{} & \multicolumn{5}{c|}{ Satisfaction vis-à-vis de l'expérience de visite } \\
\cline { 3 - 7 } \multicolumn{2}{c|}{} & $\begin{array}{c}\text { Très } \\
\text { insatisfait }\end{array}$ & Insatisfait & Neutre & Satisfait & Très satisfait \\
\hline \multirow{2}{*}{$\begin{array}{l}\text { Changement } \\
\text { d'attitude à l'égard } \\
\text { de la marque }\end{array}$} & Très défavorable & $\mathbf{2 6 , 3 \%}$ & $2,1 \%$ & $0,3 \%$ & $0,1 \%$ & $0,1 \%$ \\
\cline { 2 - 7 } & Défavorable & $\mathbf{2 2 , 8 \%}$ & $16,8 \%$ & $1,1 \%$ & $0,5 \%$ & $0,1 \%$ \\
\cline { 2 - 7 } & Neutre & $40,3 \%$ & $72,8 \%$ & $77,3 \%$ & $60,3 \%$ & $47,7 \%$ \\
\cline { 2 - 7 } & Favorable & $4,9 \%$ & $6,5 \%$ & $16,8 \%$ & $28,5 \%$ & $\mathbf{1 7 , 5 \%}$ \\
\cline { 2 - 7 } & Très favorable & $5,7 \%$ & $1,8 \%$ & $4,4 \%$ & $10,6 \%$ & $\mathbf{3 4 , 6 \%}$ \\
\hline
\end{tabular}

Note : Test du chi-deux significatif à $p<0,001$ 
Tableau 4a - Lien entre la satisfaction vis-à-vis de l'expérience de visite et l'attitude à l'égard de la marque pour les produits alimentaires et les boissons

\begin{tabular}{|l|l|c|c|c|c|c|}
\cline { 3 - 7 } \multicolumn{2}{c|}{} & \multicolumn{5}{c|}{ Satisfaction vis-à-vis de l'expérience de visite } \\
\cline { 3 - 7 } & & $\begin{array}{c}\text { Très } \\
\text { insatisfait }\end{array}$ & Insatisfait & Neutre & Satisfait & Très satisfait \\
\hline \multirow{2}{*}{$\begin{array}{l}\text { Changement } \\
\text { d'attitude à l'égard } \\
\text { de la marque }\end{array}$} & Très défavorable & $\mathbf{1 6 , 4 \%}$ & $1,7 \%$ & $0,1 \%$ & $0,1 \%$ & $0,0 \%$ \\
\cline { 2 - 7 } & Défavorable & $\mathbf{1 3 , 9 \%}$ & $11,6 \%$ & $0,7 \%$ & $0,3 \%$ & $0,1 \%$ \\
\cline { 2 - 7 } & Neutre & $\mathbf{4 8 , 4 \%}$ & $\mathbf{7 5 , 1 \%}$ & $\mathbf{7 3 , 1 \%}$ & $\mathbf{5 9 , 0 \%}$ & $\mathbf{4 6 , 8 \%}$ \\
\cline { 2 - 7 } & Favorable & $11,5 \%$ & $9,3 \%$ & $20,9 \%$ & $\mathbf{3 0 , 6 \%}$ & $\mathbf{1 8 , 0} \%$ \\
\cline { 2 - 7 } & Très favorable & $9,8 \%$ & $2,3 \%$ & $5,3 \%$ & $10,1 \%$ & $\mathbf{3 5 , 1 \%}$ \\
\hline
\end{tabular}

Note : Test du chi-deux significatif à $p<0,001$

Tableau 4b - Lien entre la satisfaction vis-à-vis de l'expérience de visite et l'attitude à l'égard de la marque pour les produits d'entretien

\begin{tabular}{|c|c|c|c|c|c|c|}
\hline & & \multicolumn{5}{|c|}{ Satisfaction vis-à-vis de l’expérience de visite } \\
\hline & & $\begin{array}{c}\text { Très } \\
\text { insatisfait }\end{array}$ & Insatisfait & Neutre & Satisfait & Très satisfait \\
\hline \multirow{5}{*}{$\begin{array}{l}\text { Changement } \\
\text { d'attitude à l'égard } \\
\text { de la marque }\end{array}$} & Très défavorable & $12,1 \%$ & $1,7 \%$ & $0,0 \%$ & $0,1 \%$ & $0,0 \%$ \\
\hline & Défavorable & $12,1 \%$ & $10,2 \%$ & $0,2 \%$ & $0,2 \%$ & $0,0 \%$ \\
\hline & Neutre & $51,5 \%$ & $62,7 \%$ & $66,0 \%$ & $49,3 \%$ & $41,7 \%$ \\
\hline & Favorable & $21,2 \%$ & $22,0 \%$ & $27,5 \%$ & $39,1 \%$ & $21,5 \%$ \\
\hline & Très favorable & $3,0 \%$ & $3,4 \%$ & $6,5 \%$ & $11,4 \%$ & $36,8 \%$ \\
\hline
\end{tabular}

Note : Test du chi-deux significatif à $p<0,001$ 
Tableau 5 - Lien entre la satisfaction et l'intention de visiter à nouveau le site (internautes visitant le site de marque pour la $1^{\text {ière }}$ fois)

\begin{tabular}{|c|c|c|c|c|c|c|}
\cline { 3 - 7 } \multicolumn{2}{c|}{} & \multicolumn{3}{c|}{ Satisfaction vis-à-vis de l'expérience de visite } \\
\cline { 3 - 7 } & $\begin{array}{c}\text { Très } \\
\text { insatisfait }\end{array}$ & Insatisfait & Neutre & Satisfait & Très satisfait \\
\hline \multirow{2}{*}{$\begin{array}{l}\text { Intention de visiter le } \\
\text { site à nouveau }\end{array}$} & Très peu probable & $\mathbf{2 9 , 0} \%$ & $3,5 \%$ & $0,8 \%$ & $0,2 \%$ & $0,3 \%$ \\
\cline { 2 - 7 } & Peu probable & $\mathbf{2 9 , 5 \%}$ & $22,3 \%$ & $5,6 \%$ & $0,9 \%$ & $0,6 \%$ \\
\cline { 2 - 7 } & Neutre & $15,6 \%$ & $33,4 \%$ & $31,5 \%$ & $10,0 \%$ & $4,7 \%$ \\
\cline { 2 - 7 } & Probable & $13,6 \%$ & $28,5 \%$ & $46,6 \%$ & $48,1 \%$ & $\mathbf{2 6 , 2} \%$ \\
\cline { 2 - 7 } & Très probable & $12,3 \%$ & $12,2 \%$ & $15,5 \%$ & $40,8 \%$ & $\mathbf{6 8 , 2} \%$ \\
\hline
\end{tabular}

Note : Test du chi-deux significatif à $p<0,001$ 
Tableau 6a - Lien entre la satisfaction vis-à-vis du contenu et la satisfaction globale (internautes visitant le site de marque pour la $1^{\text {ière }}$ fois)

\begin{tabular}{|c|l|c|c|c|c|c|}
\cline { 3 - 7 } \multicolumn{2}{c|}{} & \multicolumn{4}{|c|}{ Satisfaction globale vis-à-vis de l'expérience de visite } \\
\cline { 3 - 7 } \multicolumn{2}{c|}{} & $\begin{array}{c}\text { Très } \\
\text { insatisfait }\end{array}$ & Insatisfait & Neutre & Satisfait & $\begin{array}{c}\text { Très } \\
\text { satisfait }\end{array}$ \\
\hline \multirow{2}{*}{$\begin{array}{l}\text { Satisfaction vis-à-vis } \\
\text { du contenu proposé } \\
\text { sur le site }\end{array}$} & Très insatisfait & $\mathbf{3 8 , 2 \%}$ & $10,4 \%$ & $1,1 \%$ & $0,6 \%$ & $0,7 \%$ \\
\cline { 2 - 7 } & Insatisfait & $\mathbf{1 0 , 9 \%}$ & $26,8 \%$ & $6,6 \%$ & $5,5 \%$ & $1,2 \%$ \\
\cline { 2 - 7 } & Neutre & $\mathbf{2 3 , 0 \%}$ & $31,4 \%$ & $48,2 \%$ & $23,0 \%$ & $10,7 \%$ \\
\cline { 2 - 7 } & Satisfait & $14,6 \%$ & $24,9 \%$ & $33,7 \%$ & $52,7 \%$ & $\mathbf{3 3 , 6 \%}$ \\
\cline { 2 - 7 } & Très satisfait & $13,4 \%$ & $6,6 \%$ & $10,4 \%$ & $18,3 \%$ & $\mathbf{5 3 , 8 \%}$ \\
\hline
\end{tabular}

Note : Test du chi-deux significatif à $p<0,001$

Tableau 6b - Lien entre la satisfaction vis-à-vis de la navigation et la satisfaction globale (internautes visitant le site de marque pour la $1^{\text {ière }}$ fois)

\begin{tabular}{|c|l|c|c|c|c|c|}
\cline { 3 - 7 } \multicolumn{2}{c|}{} & \multicolumn{4}{|c|}{ Satisfaction globale vis-à-vis de l'expérience de visite } \\
\cline { 3 - 7 } \multicolumn{2}{c|}{} & $\begin{array}{c}\text { Très } \\
\text { insatisfait }\end{array}$ & Insatisfait & Neutre & Satisfait & $\begin{array}{c}\text { Très } \\
\text { satisfait }\end{array}$ \\
\hline \multirow{2}{*}{$\begin{array}{l}\text { Satisfaction vis-à-vis } \\
\text { de la navigation sur le } \\
\text { site }\end{array}$} & Très insatisfait & $\mathbf{3 3 , 9 \%}$ & $10,4 \%$ & $1,4 \%$ & $0,8 \%$ & $0,8 \%$ \\
\cline { 2 - 7 } & Insatisfait & $\mathbf{9 , 6 \%}$ & $23,2 \%$ & $7,0 \%$ & $4,8 \%$ & $1,2 \%$ \\
\cline { 2 - 7 } & Neutre & $\mathbf{2 1 , 7 \%}$ & $25,5 \%$ & $35,2 \%$ & $17,5 \%$ & $8,7 \%$ \\
\cline { 2 - 7 } & Satisfait & $\mathbf{1 7 , 7 \%}$ & $26,5 \%$ & $36,0 \%$ & $47,9 \%$ & $\mathbf{2 6 , 3 \%}$ \\
\cline { 2 - 7 } & Très satisfait & $\mathbf{1 7 , 1 \%}$ & $14,3 \%$ & $20,5 \%$ & $29,0 \%$ & $\mathbf{6 3 , 0 \%}$ \\
\hline
\end{tabular}

Note : Test du chi-deux significatif à $p<0,001$ 


\section{Encadré 1 - La solution de mesure SiteCRM ${ }^{\circledR}$}

SiteCRM, solution de mesure de la qualité de l'expérience client sur les sites de marque, utilise une approche de « test / contrôle » qui permet de mesurer l'impact du site sur la marque. L'échantillon de contrôle est constitué de premiers visiteurs invités à répondre à un questionnaire via une fenêtre pop up d'invitation présentée de façon aléatoire en entrée de site. Ce questionnaire d’une durée inférieure à cinq minutes permet d’identifier le profil du visiteur : caractéristiques socio-démographiques, relation à la marque, intention d'achat, degré de leadership dans la catégorie. L'échantillon «test» est constitué en sortie de site, via une fenêtre pop up d'invitation présentée de façon aléatoire. Le visiteur répond alors à des questions qui permettent de cerner son profil, ses raisons de visite, l'origine de sa visite (publicité online, moteur de recherche, etc.), son expérience d'ensemble sur le site, sa perception du contenu, de la navigation, de l'apparence, etc., son intention d'achat et sa perception de la marque après visite du site.

Un tableau de bord marketing disponible en ligne - un extrait d'écran de visualisation de la plateforme EzViews est présenté ci-dessous - permet à l'annonceur de suivre la qualité d'expérience des visiteurs de son site et l’impact de cette visite. En comparant les résultas des échantillons «test » et «contrôle », le responsable marketing peut apprécier l'impact de la visite sur la marque et mieux comprendre comment l'expérience et la perception du site influence la perception de la marque. Une comparaison des résultats du site à la norme sectorielle permet de mieux apprécier les performances du site et d'envisager des pistes d’améliorations.

Dans sa version Premium, la solution SiteCRM permet également de relier, visiteur par visiteur, un parcours de visite sur le site à la qualité perçue de cette visite et à son impact sur la marque. Cette option permet d'optimiser le contenu d'un site en fonction des sections / pages les plus performantes. Dans ce cas, il est alors possible de relier un comportement réel de visite (détail des pages, des sections visitées, de la durée de la visite, du temps passé par page) à la perception du site et son impact sur la relation à la marque.

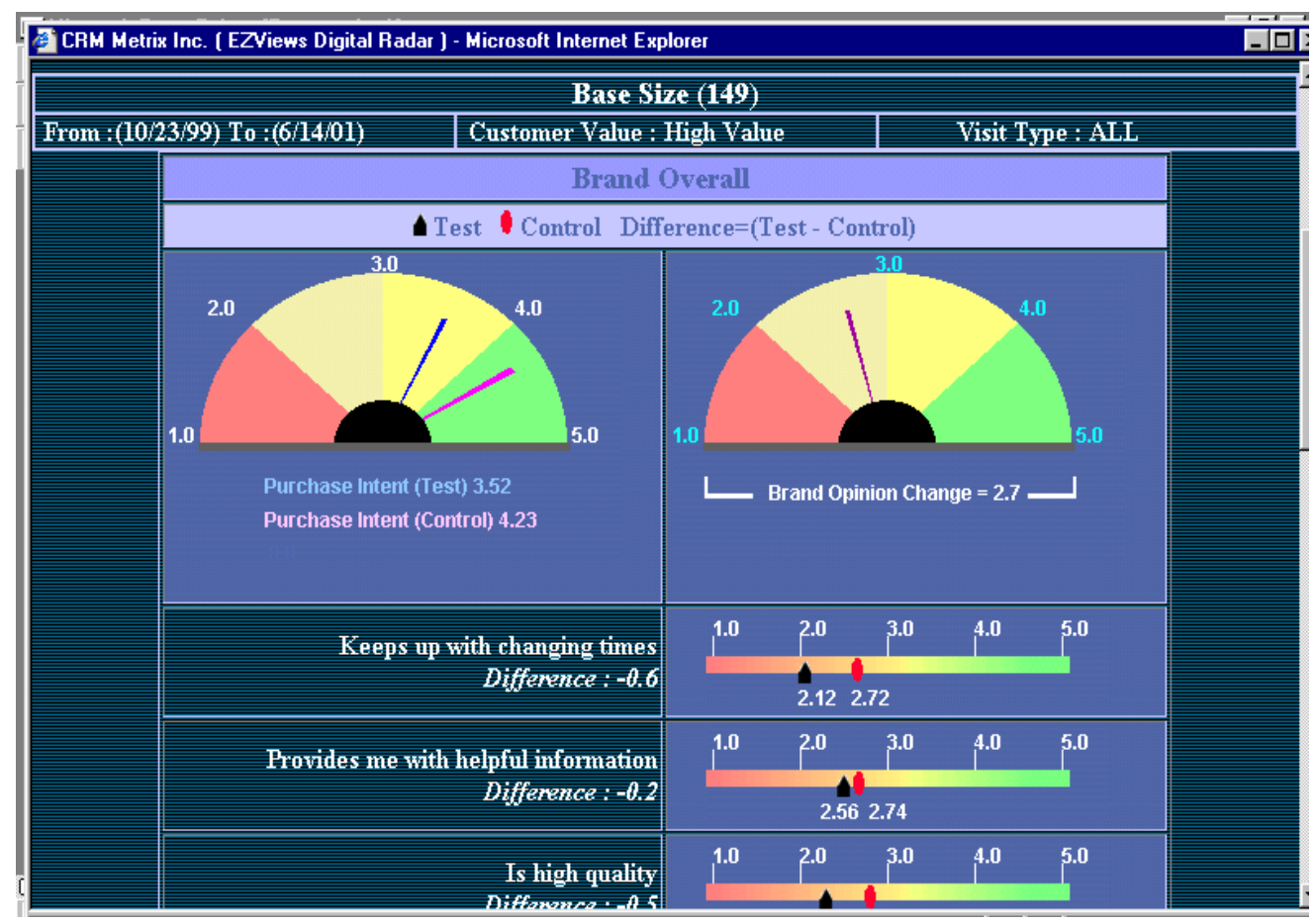




\section{Encadré 2 - Les facteurs qui déterminent la fidélité de l’internaute}

Les recherches académiques menées sur les sites marchands montrent que les leviers pour créer le trafic sont différents des leviers pour fidéliser les visiteurs. L'importance du prix, notamment, doit être fortement relativisée. Ainsi, à partir d'une étude menée auprès de plus d'un million de visiteurs du site bizrate.com (le principal guide d'achat américain), le prix apparaît clairement comme le premier facteur explicatif du trafic (10). Cependant, d'autres facteurs apparaissent comme nettement plus importants pour expliquer la probabilité de réachat : le service client (corrélation à 0,60), les délais de livraison $(0,55)$, la qualité de l'information produit $(0,50)$ et la politique de respect de la vie privée $(0,50)$. Dans cette étude, le prix arrive en dernière position pour expliquer la probabilité d'achat future (corrélation de 0,15 ). Une autre étude, menée auprès de 1211 panélistes en ligne, met en évidence que parmi les huit facteurs étudiés, le choix offert constitue le premier facteur explicatif de la fidélité, suivi de l'attractivité (site qualifié de fun, inviting, appealing...), puis de facteurs relatifs au service client et à la personnalisation (12). 


\section{Encadré 3 - Les critères pour mesurer la performance marketing des sites de marque}

Les critères de mesure de la performance d'un site de marque sont nombreux. Les critères choisis par les responsables marketing reflètent non seulement les contraintes techniques et budgétaires mais également, en théorie du moins, la vocation stratégique du site : éditoriale versus marketing ; transactionnelle / relationnelle ; conquête / fidélisation ; information / persuasion, etc.

Aujourd'hui, le nombre de visiteurs unique semble s'imposer comme le critère « généraliste » pour la plupart des sites, le chiffre d'affaires généré permettant de compléter l'analyse pour les sites marchands. Cependant, ce critère dénote clairement une logique de communication de masse. De même, le changement d'attitude vis-à-vis de la marque dénote une logique de persuasion publicitaire alors que l'intention d'achat dénote une logique transactionnelle.

D'autres critères de mesure de la performance correspondent mieux à la vocation relationnelle du site, comme la satisfaction (globale ou partielle, vis-à-vis d'éléments spécifiques de l'expérience en ligne), le pourcentage de visiteurs réguliers ou encore, l'intention de recommander le site à ses proches. En plus de ces critères relationnels principaux, de plus en plus souvent mesurés par les responsables de site, d'autres critères peuvent se révéler très pertinents, comme :

- le nombre de rubriques, de pages ou d'informations consultées... qui indique la profondeur de la visite et donc, la richesse du contenu pour la cible et les éléments de contenu les plus critiques (une étude « vu / lu » en grandeur nature) ;

- le temps passé sur le site, parfois reporté dans les études des panélistes, qui indique la présence d’un contenu attractif pour les visiteurs (à condition d'écarter les autres interprétations - comme la difficulté à trouver ce que l'on cherche - et à contourner les problèmes techniques de mesure - comme les sessions interrompues) ;

- le pourcentage de visiteurs qui s'enregistrent sur le site ou qui répondent aux études en ligne proposées, qui indique une volonté de poursuivre la relation au-delà d'une simple visite (notion d'engagement relationnel et de coopération).

Il est important de construire progressivement des données normatives, issues des réponses aux mêmes questions posées de façon constante sur plusieurs périodes. Ces normes deviennent plus fiables et plus précises au cours du temps : elles permettent notamment des analyses par segments de clients. Ces normes sont utiles pour étudier l'évolution des performances du site dans le temps et pour comparer les sites entre eux (benchmarking). Typiquement une analyse des « drivers » de l'expérience en ligne peut, par exemple, permettre à un responsable de site de mettre en place les priorités d'améliorations du site et d'en mesurer les effets après la mise en place d'un nouveau design.

Aujourd'hui, les logiciels d'analyse du trafic permettent de répondre en partie à ces interrogations. Toutefois, ces logiciels ne donnent qu'une petite partie des informations nécessaires pour améliorer l'expérience en ligne, voire même, pour engager une conversation avec le visiteur. Certes, on connaît les comportements sur le site, les produits passés en revue et les informations consultées (le "quoi»), mais on ignore complètement les motivations et les attitudes (le « pourquoi »). La mise en place de questionnaires en sortie de site permet donc de compléter les données issues des logiciels d'analyse comportementales. 
Figure 1 - Le site de marque, un moyen pour entrer en relation avec les meilleurs clients de la marque (cas d'une marque de PGC américaine extrait de la base de données SiteCRM)

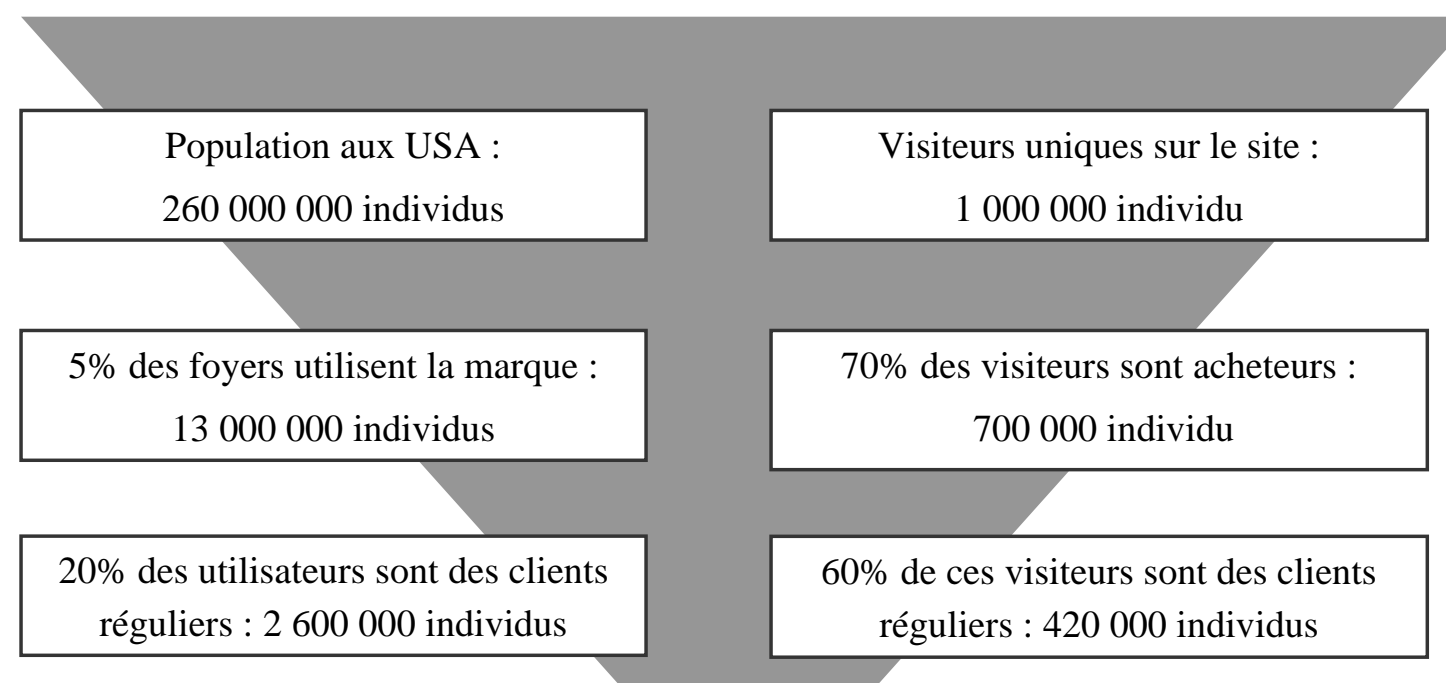

$0,4 \%$ du total

$5,5 \%$ du total

$16 \%$ du total 